\title{
Comparison of HIF1A-AS1 and HIF1A-AS2 in regulating HIF-1 $\alpha$ and the osteogenic differentiation of PDLCs under hypoxia
}

\author{
DONGRU CHEN ${ }^{1}$, LIPING WU ${ }^{1}$, LU LIU $^{2}$, QIMEI GONG ${ }^{2}$, \\ JINXUAN ZHENG ${ }^{1}$, CAIXIA PENG ${ }^{1}$ and JIANQING DENG ${ }^{1}$
}

\author{
Departments of ${ }^{1}$ Orthodontics and ${ }^{2}$ Endodontics, Guanghua School of Stomatology, Guangdong Provincial \\ Key Laboratory of Stomatology, Sun Yat-sen University, Guangzhou, Guangdong 510055, P.R. China
}

Received August 19, 2016; Accepted August 29, 2017

DOI: $10.3892 /$ ijmm.2017.3138

\begin{abstract}
Hypoxia-inducible factor- $1 \alpha(\mathrm{HIF}-1 \alpha)$ is essential for regulating the osteogenic differentiation of periodontal ligament cells (PDLCs). The regulatory mechanism of HIF-1 $\alpha$ transcription is still not clear. Recently, two long non-coding RNAs, HIF1A antisense RNA 1 (HIF1A-AS1) and HIF1A antisense RNA 2 (HIF1A-AS2), were found to regulate HIF-1 $\alpha$ mRNA, but the regulatory mechanisms among HIF-1 $\alpha$, HIF1A-AS1 and HIF1A-AS2 have not been well studied. We hypothesized that HIF1A-AS1 and HIF1A-AS2 play important roles in the osteogenic differentiation of PDLCs by regulating HIF-1 $\alpha$. In the present study, we showed that expression levels of HIF1A-AS1, HIF1A-AS2, HIF-1 $\alpha$ and osteogenic biomarkers were time-dependent under hypoxia. Even though both HIF1A-AS1 and HIF1A-AS2 were complementary to HIF-1 $\alpha$ mRNA, only HIF1A-AS2 showed an inhibitory effect on HIF-1 $\alpha$ in PDLCs. Moreover, HIF-1 $\alpha$ had positive regulatory effects on HIF1A-AS1 and HIF1A-AS2. HIF-1 $\alpha$ promoted the osteogenic differentiation of PDLCs, and HIF1A-AS2 had a negative effect on the osteogenic differentiation of PDLCs. Altogether, the present study revealed the complex relationships among HIF1A-AS1, HIF1A-AS2 and HIF-1 $\alpha$, as well as their roles in regulating the osteogenic differentiation of PDLCs. These findings provide a theoretical basis for promoting periodontal tissue regeneration and repair during orthodontic tooth movement.
\end{abstract}

\section{Introduction}

Periodontitis and malocclusion along with caries are significant public oral diseases with extremely high incidence

Correspondence to: Dr Liping Wu, Department of Orthodontics, Guanghua School of Stomatology, Guangdong Provincial Key Laboratory of Stomatology, Sun Yat-sen University, 56 Lingyuanxi Road, Guangzhou, Guangdong 510055, P.R. China

E-mail: wulping@mail.sysu.edu.cn

Key words: hypoxia, hypoxia-inducible factor-1 $\alpha$, HIF1A antisense RNA 1, HIF1A antisense RNA 2, osteogenic differentiation, periodontal ligament cells rates. Epidemiologic studies show that more than $50 \%$ of adults suffer from periodontitis and severe periodontitis is estimated to occur in approximately $5-20 \%$ of adults worldwide (1-3). The prevalence of malocclusion among children is also more than $50 \%$. These two oral diseases are major public health issues that require attention. Periodontal ligament (PDL) is a complex tissue with abundant blood vessels and cells, including periodontal ligament cells (PDLCs), periodontal ligament stem cells, fibroblasts, osteoblasts and osteoclasts, which play a vital role in periodontal tissue regeneration (4). Periodontitis and orthodontic tooth movement can cause hypoxia around the PDL, which triggers a series of molecular responses in PDLCs for hypoxic adaptation (4). The most sensitive and important molecule is functional hypoxia-inducible factor-1 (HIF-1), composed of HIF- $\alpha$ (HIF-1 $\alpha, \mathrm{HIF}-2 \alpha$ and HIF-3 $\alpha$ ) and HIF- $\beta$ subunits. HIF- $1 \alpha$ is the dominant functional subunit that is unstable and tends to be degraded under normoxia, but it can be highly increased in short-term hypoxia $(5,6)$. After translocating into the nucleus and dimerizing with HIF- $\beta$, HIF-1 $\alpha$ binds to hypoxia response elements (HREs) of $>100$ genes and regulates their production, such as vascular endothelial growth factor (VEGF), transforming growth factor- $\beta$, erythropoietin (EPO) and microRNAs $(7,8)$. Meanwhile, HIF-1 $\alpha$ has been confirmed to regulate osteogenic differentiation, yet there is still controversy over whether HIF-1 $\alpha$ promotes or inhibits osteogenic differentiation (9-12).

Since HIF-1 $\alpha$ was discovered, its regulatory mechanism has aroused much interest. It is known that HIF-1 $\alpha$ protein can be rapidly degraded under normoxia by the Von Hippel-Lindau protein-mediated ubiquitin-proteasome pathway. The degradation of HIF-1 $\alpha$ protein is regulated by many factors, such as mitogen-activated protein kinase (MAPK) and phosphatidylinositol 3 kinase (13-16). However, the regulatory mechanism of HIF- $1 \alpha$ mRNA has not been well studied. Thrash-Bingham and Tartof (17) firstly named an antisense non-coding RNA as aHIF that originated from the 3' region of the hif- $1 \alpha$ gene and could bind to the HIF-1 $\alpha$ mRNA $3^{\prime}$ untranslated region (UTR), and aHIF was also named as HIF1A antisense RNA 2 (HIF1A-AS2) or $3{ }^{\prime} \alpha H I F-1 \alpha$. Then in 2010, Baranello et al (18) identified another antisense non-coding RNA that originated from the 5' region of the hif- $1 \alpha$ gene, named HIF1A-AS1 or 5'aHIF-1 $\alpha$. Both HIF1A-AS1 and HIF1A-AS2 are longer than $200 \mathrm{nt}$ and belong to the family 
of long non-coding RNAs (lncRNAs). Yet, they are different in structure and loci. HIF1A-AS1 has both 5'cap and poly (A+) tail, while HIF1A-AS2 has neither of them. HIF1A-AS1 accumulates at the nuclear membrane, while HIF1A-AS2 locates only in the nucleus (19).

HIF1A-AS2 can downregulate HIF-1 $\alpha$ mRNA so that during long-term hypoxia HIF-1 $\alpha$ protein is suppressed (20). Moreover, the putative HIF-1 $\alpha$ protein binding sites-HREs are found in the HIF1A-AS2 promoter region by analyzing its RNA sequence, which indicates that HIF-1 $\alpha$ might also regulate HIF1A-AS2, but this is not yet confirmed (21). Overall, HIF1A-AS2 and HIF-1 $\alpha$ have a complicated regulatory mechanism. For the first time, we demonstrated that HIF1A-AS1 and HIF1A-AS2 exist in PDLCs. We then explored the differences between HIF1A-AS1 and HIF1A-AS2 in regulating HIF-1 $\alpha$ and the osteogenic differentiation of PDLCs under hypoxia. Runt-related transcription factor 2 (Runx2) is a key factor initiating and regulating the early osteogenesis and late mineralization of bone (22). Alkaline phosphatase (ALP) activity can also describe the early cell differentiation of osteoblastic cells (23). Therefore, Runx 2 and ALP activity were selected as the osteogenic biomarkers in the present study. Given that the osteogenic differentiation mechanism in PDLCs is essential to periodontal tissue regeneration, the present research can provide a theoretical basis for promoting periodontal tissue remodeling, regeneration and repair during orthodontic tooth movement and periodontitis.

\section{Materials and methods}

Bioinformatic analysis. UCSC Genome Bioinformatics (http://genome.ucsc.edu/) was used to locate the HIF-1 $\alpha$, HIF1A-AS1 and HIF1A-AS2 genes, as well as to obtain HIF-1 $\alpha$ mRNA, HIF1A-AS1 RNA and HIF1A-AS2 RNA sequences. In addition, we used the basic local alignment search tool to obtain the complementary regions between HIF1A-AS1 and HIF-1 $\alpha$ mRNA, as well as HIF1A-AS2 and HIF- $1 \alpha$ mRNA. Furthermore, we examined the promoter regions of HIF1A-AS1 and HIF1A-AS2 to determine whether they have putative HRE sequences.

Sample collection and cell culture. Healthy premolars or third-molars were collected from patients $(<25$ years of age) at the Hospital of Stomatology, Sun Yat-sen University. Informed consent from each patient was obtained, and the present study was approved by the Ethics Committee of the Hospital of Stomatology, Sun Yat-sen University. PDL was separated from the middle-third of the root surface, washed with phosphate-buffered saline (PBS; Life Technologies, Grand Island, NY, USA), digested using type I collagenases (Life Technologies), and then cultured in primary culture medium containing 78\% Modified Eagle's Medium $\alpha(\mathrm{MEM} \alpha), 20 \%$ fetal bovine serum (FBS) (both from Gibco BRL, Gaithersburg, MD, USA), and 2\% antibiotics. Cells passaged to $\mathrm{P}_{4}$ were used in the following experiments. For the hypoxia group, cells were incubated in a three-air hypoxia chamber (Galaxy 170R; Eppendorf Co., Ltd., Hamburg, Germany) with $2 \% \mathrm{O}_{2}, 5 \% \mathrm{CO}_{2}$ and $93 \% \mathrm{~N}_{2}$ at $37^{\circ} \mathrm{C}$, while the normoxia group was incubated in a chamber with $20 \% \mathrm{O}_{2}$,
Table I. Primer sequences used in this study.

\begin{tabular}{llc}
\hline Gene name & \multicolumn{1}{c}{$\begin{array}{c}\text { Primer sequence } \\
\text { (forward and reverse) }\end{array}$} & $\begin{array}{c}\text { Product } \\
\text { size (bp) }\end{array}$ \\
\hline HIF1A-AS1 (F) & 5'-AGGCAGAGACGAGATGAACA-3' & 100 \\
HIF1A-AS1 (R) & 5'-AGGCAGAGACGAGATGAACA-3' & \\
HIF1A-AS1 (RT) & 5'-CTGGGTCTGGCCATTTCATT-3' & \\
HIF1A-AS2 (F) & 5'-GACCTAAGGCTCTGGCACTT-3' & \multirow{2}{*}{80} \\
HIF1A-AS2 (R) & 5'-CACTATGAATCCCTGCACCT 3' & \\
HIF1A-AS2 (RT) & 5'-CACTATGAATCCCTGCACCT-3' & \\
U6 F & 5'-CTCGCTTCGGCAGCACA-3' & \multirow{2}{*}{94} \\
U6 R & 5'-AACGCTTCACGAATTTGCGT-3' & \\
U6 RT & 5'-AACGCTTCACGAATTTGCGT-3' & \\
HIF-1 $\alpha$ & 5'-GTGGATTACCACAGCTGA-3' & 115 \\
& 5'-GCTCAGTTAACTTGATCCA-3' & \\
Runx2 & 5'-TCTAAATCGCCAGGCTTCAT-3' & 250 \\
& 5'-GAGGACCTACTCCCAAAGGA-3' & \\
3-actin & 5'-TGGATCAGCAAGCAGGAGTA-3' & 275 \\
& 5'-TCGGCCACATTGTGAACTTT-3' &
\end{tabular}

HIF1A-AS1, HIF1A antisense RNA 1; HIF1A-AS2, HIF1A antisense RNA 2; HIF-1 $\alpha$, hypoxia-inducible factor- $1 \alpha$; Runx2, runt-related transcription factor 2 .

$5 \% \mathrm{CO}_{2}$ and $75 \% \mathrm{~N}_{2}$ at $37^{\circ} \mathrm{C}$ (Shellab 2323-2; Shellab, Cornelius, OR, USA). Each group was divided into four subgroups $(6,12,24$ and $48 \mathrm{~h})$.

Reverse transcription-quantitative reverse transcriptase polymerase chain reaction $(R T-q P C R)$. Total RNA was extracted from PDLCs using TRIzol reagent (Life Technologies) at each time point. The quality and quantity of RNA were measured. Total RNA $(1.0 \mu \mathrm{g})$ was reverse transcribed for cDNA synthesis (ImProm-II ${ }^{\mathrm{TM}}$ Reverse Transcription system; Promega Corp., Madison, WI, USA). Then cDNA was mixed with 2X SYBR-Green master mix (Life Technologies) and gene-specific primers in a final volume of $20 \mu \mathrm{l}$, and the realtime PCR was performed on the LightCycler $\AA^{\circledR} 480$ platform (Roche, Basel, Switzerland) with the following reaction: $50^{\circ} \mathrm{C}$ for $2 \mathrm{~min}$ and $95^{\circ} \mathrm{C}$ for $2 \mathrm{~min}$, followed by 40 cycles at $95^{\circ} \mathrm{C}$ for $15 \mathrm{sec}$ and $60^{\circ} \mathrm{C}$ for $32 \mathrm{sec}$. The primers used in the present study are listed in Table I. U6 snRNA was used to normalize the expression level of HIF1A-AS1 and HIF1A-AS2, and $\beta$-actin was used to normalize the expression level of HIF-1 $\alpha$ and Runx2.

Western blot analysis. PDLCs under $2 \%$ and $20 \% \mathrm{O}_{2}$ at each time point were lysed using lysis buffer (99\% RIPA, 1\% PMSF) and the protein concentration was determined by a bicinchoninic acid (BCA) protein assay kit (CWBio, Co., Ltd., Beijing, China). Equal amount of protein was separated by electrophoresis on duplicate $10 \%$ sodium dodecyl sulphate-polyacrylamide gel electrophoresis (SDS-PAGE) gels, then transferred to polyvinylidene fluoride membranes and incubated with primary antibodies, mouse anti-human HIF-1 $\alpha$ (1:1,000 dilution; cat. no. ab113642; Abcam, Cambridge, MA, USA) and 
Table II. Sequences of the siRNAs used in this study.

\begin{tabular}{llc}
\hline $\begin{array}{l}\text { Gene name/ } \\
\text { siRNA }\end{array}$ & \multicolumn{1}{c}{ Sequences $\left(5^{\prime} \rightarrow 3^{\prime}\right)$} & $\begin{array}{c}\text { Size } \\
(\mathrm{bp})\end{array}$ \\
\hline $\begin{array}{l}\text { HIF1A-AS1 } \\
\text { siRNA1-sense }\end{array}$ & AUGGCCAGACCCAGAUGUUdTdT & 21 \\
siRNA1-antisense & AACAUCUGGGUCUGGCCAUdTdT & \\
siRNA2-sense & GCUCCAGAACGCAGAGGAAdTdT & 21 \\
siRNA2-antisense & UUCCUCUGCGUUCUGGAGCdTdT & \\
HIF1A-AS2 & & \\
siRNA1-sense & CUUAAAUUGUUGGUAAACAdTdT & 21 \\
siRNA1-antisense & UGUUUACCAACAAUUUAAGdTd & \\
siRNA2-sense & GUAACAUUGUGACUAUAAUdTd & 21 \\
siRNA2-antisense & AUUAUAGUCACAAUGUUACdTdT & \\
HIF-1 $\alpha$ & & \\
siRNA1-sense & CAAAGUUCACCUGAGCCUAdTdT & 21 \\
siRNA1-antisense & UAGGCUCAGGUGAACUUUGdTT & \\
siRNA2-sense & GAUUAACUCAGUUUGAACUdTdT & 21 \\
siRNA2-antisense & AGUUCAAACUGAGUUAAUCdTdT & \\
NC & & \\
siRNA-sense & UUCUCCGAACGUGUCACGUTT & 21 \\
siRNA-antisense & ACGUGACACGUUCGGAGAATT & \\
\hline siRNA, small interfering RNA; HIF1A-AS1, HIF1A antisense RNA 1; & \\
HIF1A-AS2, HIF1A & antisense RNA 2; HIF-1 $\alpha$, hypoxia-inducible \\
factor-1 $\alpha$; NC, non-specific control. & \\
\hline
\end{tabular}

rabbit anti-human Runx2 (1:1,000 dilution; cat. no. 12556; Cell Signaling Technology, Inc., Danvers, MA, USA), for $24 \mathrm{~h}$ at $4^{\circ} \mathrm{C}$. After washing, the membranes were incubated with the corresponding secondary antibodies (anti-mouse IgG, cat. no. M281; anti-rabbit IgG, cat. no. M283; both from Takara Bio, Inc., Otsu, Japan) for $1 \mathrm{~h}$ at $37^{\circ} \mathrm{C}$. Chemiluminescence was imaged and then the band intensity was quantified by ImageJ software [National Institutes of Health (NIH), Bethesda, MD, USA]. The relative protein levels were calculated as the ratio to the level of $\beta$-actin.

ALP activity. PDLCs were cultured in 6-well plates in 2 or $20 \% \mathrm{O}_{2}$. Cells were lysed by Triton X-100 (1\% Triton X-100, 99\% PBS). The protein concentration was determined using the BCA protein assay kit. The supernatant was then used for the ALP assay (Nanjing Jiancheng Bioengineering Institute, Nanjing, China), and the absorbance was measured at a wavelength of $520 \mathrm{~nm}$. The ALP activity was determined according to the manufacturer's instructions.

Transfection of small interfering RNAs (siRNAs). siRNAs targeting HIF1A-AS1, HIF1A-AS2, HIF-1 $\alpha$ and non-specific control siRNAs (NC si) were designed by Sigma (St. Louis, MO, USA). NC si was used as a negative control. The sequences are listed in Table II. siRNA was dissolved to a concentration of $20 \mu \mathrm{M}$, and then HIF1A-AS1 siRNA, HIF1A-AS2 siRNA and HIF-1 $\alpha$ siRNA $(5 \mu 1)$ were transfected using Lipofectamine $^{\mathrm{TM}}$ RNAiMAX into PDLCs, respectively. In addition, HIF1A-AS1 siRNA $(2.5 \mu \mathrm{l})$ and HIF1A-AS2 siRNA
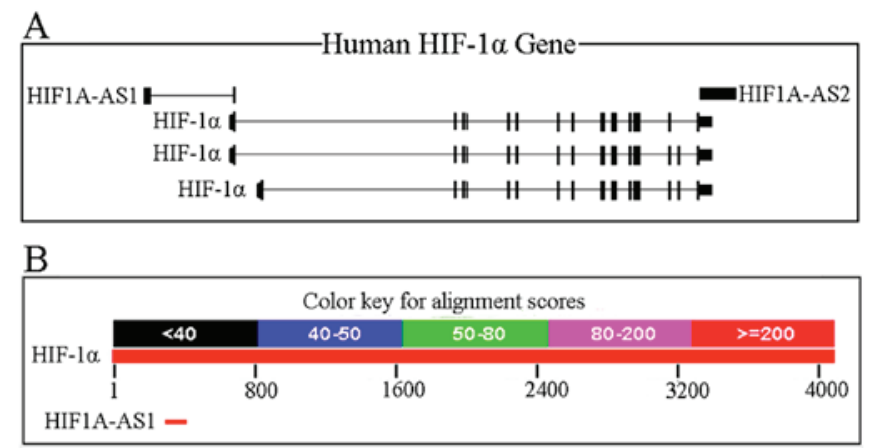

\begin{tabular}{lccc} 
Range: 1 to 117 & & \\
Score & Identities & Gaps & \\
217 bits (117) & $117 / 117(100 \%)$ & $0 / 117(0 \%)$ & \\
\hline HIF-1a & 307 & CTTTCCTTCTCTTCT......CCAAGGGCGCCGGCGGCG & 423 \\
HIF1A-AS1 & 117 & CTTTCCTTCTCTTCT......CCAAGGGCGCCGGCGGCG & 1 \\
\hline
\end{tabular}

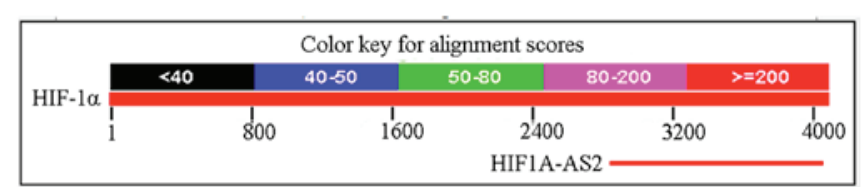

\begin{tabular}{lllll}
\multicolumn{2}{l}{\begin{tabular}{l} 
Range: 831 to 2051 \\
\hline Score
\end{tabular}} & \multicolumn{4}{l}{ Identities } & Gaps \\
2255 bits (1221) & $1221 / 1221(100 \%)$ & $0 / 1221(0 \%)$ & \\
\hline HIF-1 $\alpha$ & 2839 & TACTGCAGGGTGAAG......ATCTTCTGTGGACCAGGC & 4059 \\
HIIF1A-AS2 & 2051 & TACTGCAGGGTGAAG.....ATCTTCTGTGGACCAGGC & 831 \\
\hline
\end{tabular}

Figure 1. Bioinformatic analysis of HIF-1 $\alpha$, HIF1A-AS1 and HIF1A-AS2 gene and RNA. (A) Relative gene positions of human HIF-1 $\alpha$, HIF1A-AS1 and HIF1A-AS2. (B) Complementary regions and sequences of HIF1A-AS1 with HIF-1 $\alpha$ mRNA, as well as HIF1A-AS2 with HIF-1 $\alpha$ mRNA. HIF-1 $\alpha$, hypoxia-inducible factor-1 $\alpha$; HIF1A-AS1, HIF1A antisense RNA 1; HIF1A-AS2, HIF1A antisense RNA 2.

(2.5 $\mu \mathrm{l})$ were transfected at the same time to knock down both HIF1A-AS1 and HIF1A-AS2. After transfection for $48 \mathrm{~h}$, PDLCs were cultured under hypoxia for $12 \mathrm{~h}$, and then total RNA and protein were collected for RT-qPCR, western blot analysis and ALP activity.

Statistical analysis. Statistical analysis was carried out using SPSS 13.0 (SPSS, Inc., Chicago, IL, USA). All of the results are expressed as the mean \pm SEM. Data for the groups were compared by two-tailed Student's t-test and the significant level was set at $\mathrm{P}<0.05$.

\section{Results}

Bioinformatic analysis of HIF-1 $\alpha, H I F 1 A-A S 1$ and HIF1A-AS2. Gene locations for HIF1A-AS1 and HIF1A-AS2 in the human were from chr14:61681041-61695823 and chr14:61747039-61749089. HIF-1 $\alpha$ had three transcripts, in which transcript 1 and 2 were from chr14:61695401-61748259 and transcript 3 was from chr14:61697622-61748259. The relative locations of HIF-1 $\alpha$, HIF1A-AS1 and HIF1A-AS2 are shown in Fig. 1A.

The sizes of HIF1A-AS1 mRNA and HIF1A-AS2 mRNA were 652 and 2,051 nt. As shown in Fig. 1B, HIF1A-AS1 was complementary upon $117 \mathrm{nt}$ to the HIF-1 $\alpha$ mRNA 5'UTR (HIF1A-AS1: 117 to 1, HIF-1 $\alpha$ mRNA: 307 to 423), while HIF1A-AS2 was complementary upon 1,221 nt to 


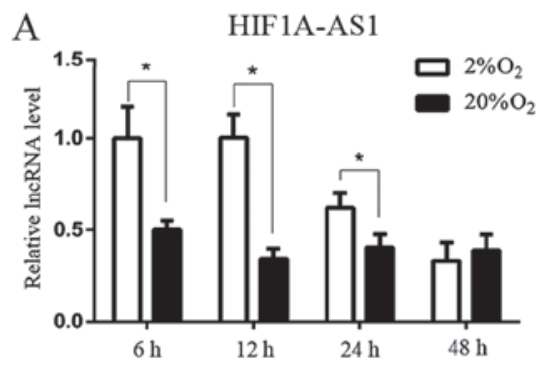

$\mathrm{D}$

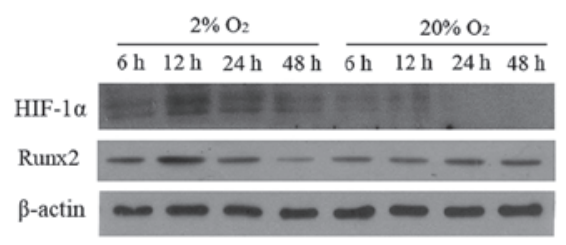

B

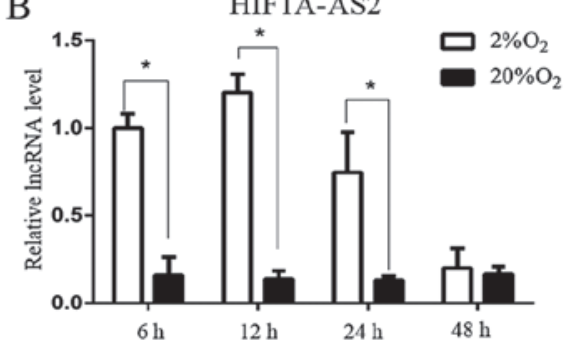

E

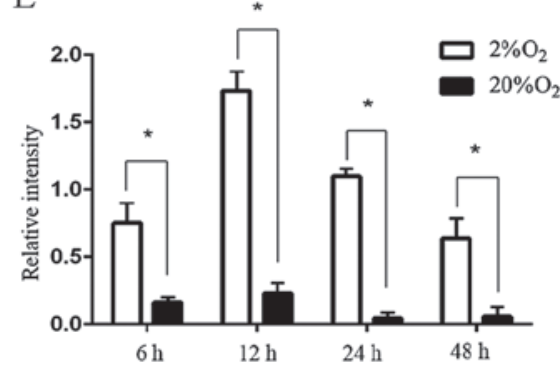

C HIF- $1 \alpha$ mRNA

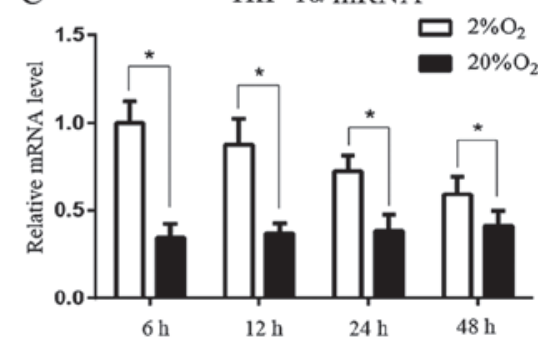

F Runx2 mRNA

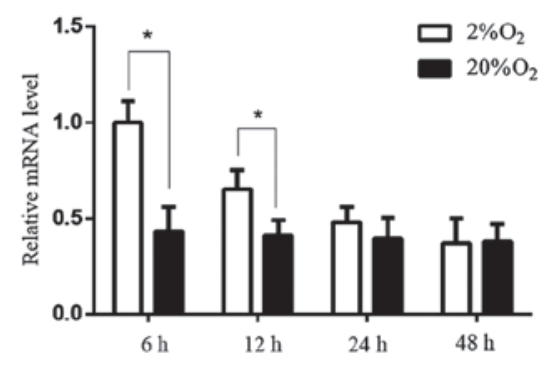

G

$\mathrm{H}$

ALP activity
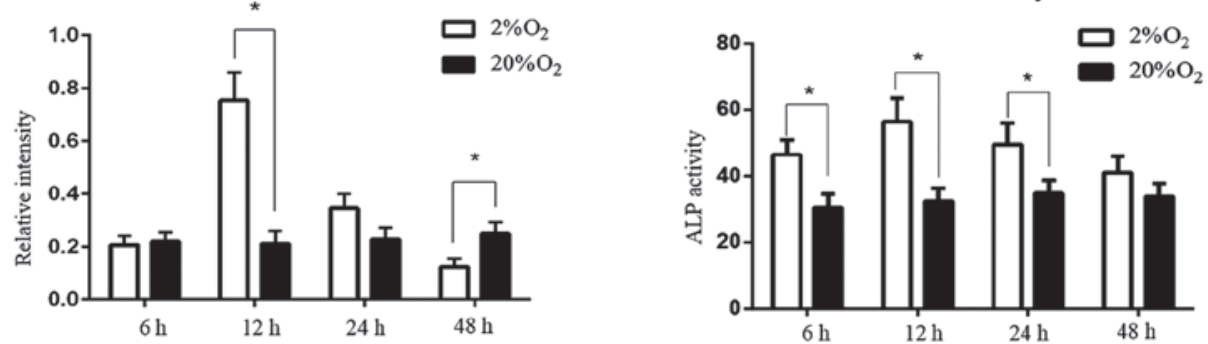

Figure 2. Expression patterns of HIF1A-AS1, HIF1A-AS2, HIF-1 $\alpha$, ALP and Runx2 in PDLCs under hypoxia and normoxia. (A and B) The lncRNA levels of HIF1A-AS1 and HIF1A-AS2 under hypoxia were significantly increased at 6, 12 and $24 \mathrm{~h}$, and peaked at $12 \mathrm{~h}$. (C) The mRNA level of HIF-1 $\alpha$ was significantly higher under hypoxia than that under normoxia and gradually decreased from 6 to $48 \mathrm{~h}$. (D and E) The protein expression of HIF-1 $\alpha$ under hypoxia was notably higher than that under normoxia, and peaked at $12 \mathrm{~h}$. (F) The mRNA level of Runx 2 was induced by hypoxia before $12 \mathrm{~h}$. (D and G) The protein expression of Runx2 under hypoxia was significantly induced at $12 \mathrm{~h}$, and then was suppressed at $48 \mathrm{~h}$. (H) The ALP activity under hypoxia was induced significantly at 6,12 and $24 \mathrm{~h}$, and peaked at $12 \mathrm{~h}$. "P<0.05 vs. the normoxia group, $\mathrm{n}=3$. HIF1A-AS1, HIF1A antisense RNA 1; HIF1A-AS2, HIF1A antisense RNA 2; HIF-1 $\alpha$, hypoxia-inducible factor-1 $\alpha$; ALP, alkaline phosphatase; Runx2, runt-related transcription factor 2; PDLCs, periodontal ligament cells.

HIF-1 $\alpha$ mRNA 3'UTR (HIF1A-AS2: 2,051 to 831, HIF-1 $\alpha$ mRNA: 2,839 to 4,059$)$. Consensus putative HRE sequence was 5'-(A/G)GTG-3', which has been identified in many hypoxia-inducible genes, such as VEGF and EPO $(24,25)$. Commonly, the promoter region was from $-2,000$ to $+200 \mathrm{bp}$ from the RNA transcription initiation site. After searching for HIF1A-AS1 and HIF1A-AS2 promoter, we identified several putative HREs within the regions (HIF1A-AS1: -194, - 492 and -661, HIF1A-AS2: -63 and -600), indicating that HIF-1 $\alpha$ may also regulate the two long non-coding RNAs.

HIF1A-AS1, HIF1A-AS2, HIF-1 $\alpha$ expression and the osteogenic differentiation of PDLCs under hypoxia at different times. Both HIF1A-AS1 and HIF1A-AS2 were expressed in the PDLCs. Hypoxia increased the level of HIF1A-AS1 to 2.00 -fold, 2.26-fold and 1.53-fold (Fig. 2A) and HIF1A-AS2 to 6.33-fold, 8.77-fold and 5.79-fold (Fig. 2B) at 6,12 and $24 \mathrm{~h}$ $(\mathrm{P}<0.05)$ in the PDLCs when compared with levels in the normoxia groups. Both HIF1A-AS1 and HIF1A-AS2 began to decrease under hypoxia after $12 \mathrm{~h}$.

HIF-1 $\alpha$ mRNA (Fig. 2C) was significantly upregulated at $6 \mathrm{~h}$, under hypoxia $(\mathrm{P}<0.05)$, but it decreased gradually from
6 to $48 \mathrm{~h}$. Meanwhile, HIF-1 $\alpha$ protein (Fig. 2D and E) was barely detectable under normoxia, but it was sharply induced under hypoxia from $6 \mathrm{~h}$, peaked at $12 \mathrm{~h}$, then gradually diminished from 12 to $48 \mathrm{~h}$, yet still higher than the normoxia groups $(\mathrm{P}<0.05)$.

The expression level of Runx 2 mRNA (Fig. 2F) under hypoxia was also higher than the level in the normoxia groups at 6 and $12 \mathrm{~h}(\mathrm{P}<0.05)$, then it gradually decreased to the baseline at 24 and $48 \mathrm{~h}(\mathrm{P}>0.05)$. Runx2 protein (Fig. $2 \mathrm{D}$ and $\mathrm{G})$ was notably induced at $12 \mathrm{~h}$ under hypoxia, peaked at $12 \mathrm{~h}$, and was suppressed at $48 \mathrm{~h}$ when compared with the normoxia group $(\mathrm{P}<0.05)$. ALP activity (Fig. $2 \mathrm{H})$ was facilitated significantly under hypoxia at 6,12 and $24 \mathrm{~h}(\mathrm{P}<0.05)$, reaching a peak at $12 \mathrm{~h}$, but showing no significant difference at $48 \mathrm{~h}$ $(\mathrm{P}>0.05)$.

Silencing efficiency of HIF1A-AS1, HIF1A-AS2 and $H I F-1 \alpha$. To further investigate the regulatory mechanism among HIF1A-AS1, HIF1A-AS2 and HIF-1 $\alpha$, we silenced HIF1A-AS1, HIF1A-AS2 and HIF-1 $\alpha$. The siRNAs of HIF1A-AS1, HIF1A-AS2, HIF-1 $\alpha$ and NC were successfully transfected into PDLCs. The transfection of NC si is 


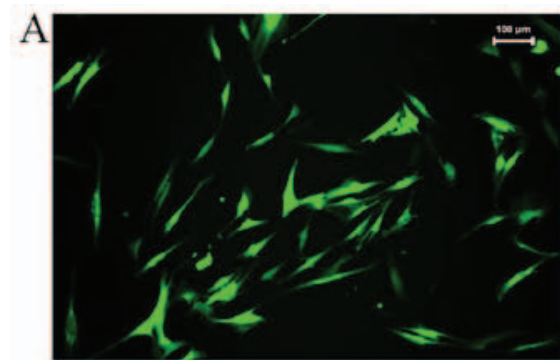

Fluorescent field

B

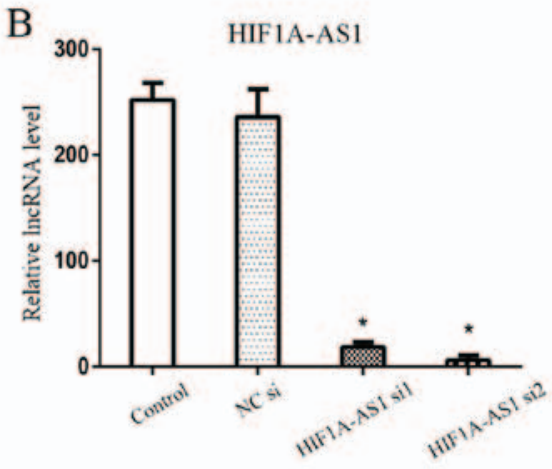

$\mathrm{C}$

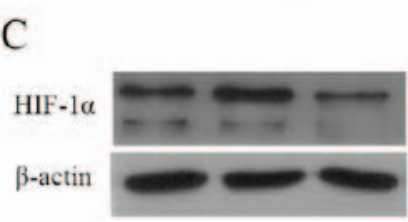

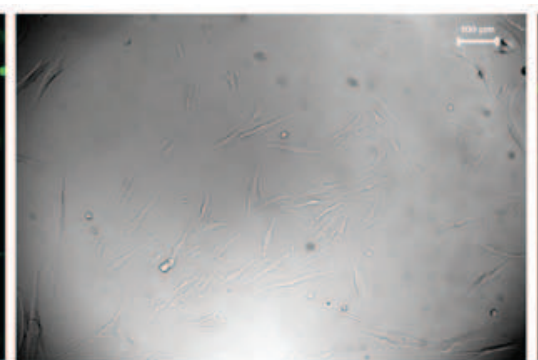

Bright field
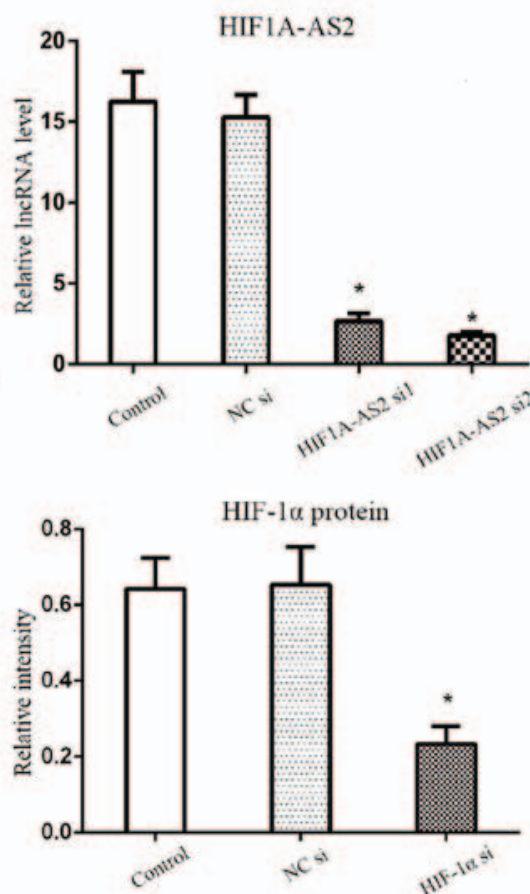
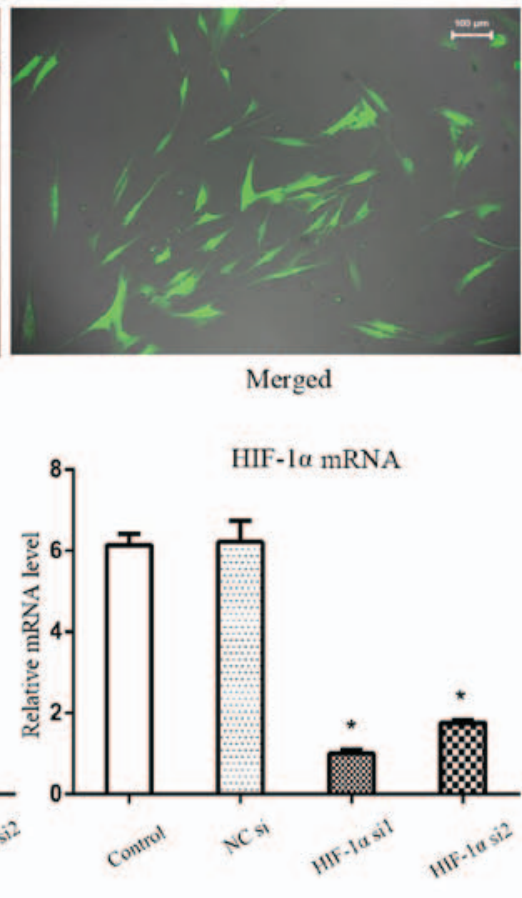

Merged

Figure 3. Silencing efficiencies of HIF1A-AS1, HIF1A-AS2 and HIF-1 $\alpha$ siRNAs. (A) NC si shown as an example was successfully transfected into PDLCs (B) HIF1A-AS1, HIF1A-AS2 and HIF-1 $\alpha$ mRNA were effectively reduced by $>90,80$ and $70 \%$ in PDLCs under hypoxia at 12 h, respectively. (C) HIF-1 protein was significantly inhibited by HIF-1 $\alpha$ siRNA in hypoxia. " $\mathrm{P}<0.05$ vs. the control group, $\mathrm{n}=3$. HIF1A-AS1, HIF1A antisense RNA 1; HIF1A-AS2, HIF1A antisense RNA 2; HIF-1 $\alpha$, hypoxia-inducible factor-1 $\alpha$; NC si, non-specific control siRNA; PDLCs, periodontal ligament cells.

shown in Fig. 3A. Knockdown efficiencies were detected by RT-qPCR for HIF1A-AS1, HIF1A-AS2 and HIF-1 $\alpha$ mRNA, and western blot analysis for HIF-1 $\alpha$ protein. Results showed that the silencing efficiencies of HIF1A-AS1, HIF1A-AS2 and HIF-1 $\alpha$ mRNA (Fig. 3B) were $>90,80$ and $70 \%$ respectively. Furthermore, HIF-1 $\alpha$ protein (Fig. 3C) was also successfully inhibited by HIF-1 $\alpha$ siRNA under hypoxia.

Effects of silencing HIF1A-AS1 and HIF1A-AS2 on HIF-1 $\alpha$ in PDLCs. Changes in HIF-1 $\alpha$ mRNA (Fig. 4A) and HIF-1 $\alpha$ protein (Fig. 4B and $\mathrm{C}$ ) were consistent following the silencing of HIF1A-AS1 and/or HIF1A-AS2. Silencing of HIF1A-AS1 did not significantly alter the expression of HIF-1 $\alpha$ protein and mRNA, whereas silencing of HIF1A-AS2 significantly induced the expression of HIF-1 $\alpha$ mRNA and protein. In order to determine whether HIF1A-AS1 and HIF1A-AS2 had synergistic effects on HIF-1 $\alpha$ expression and osteogenic differentiation, we knocked down HIF1A-AS1 and HIF1A-AS2 by co-transfecting PDLCs with HIF1A-AS1 siRNA and HIF1A-AS2 siRNA. Results showed that HIF-1 $\alpha$ protein and mRNA were significantly elevated, similar to silencing HIF1A-AS2 alone.
Effects of silencing HIF-1 on HIF1A-AS1 and HIF1A-AS2 in PDLCs. Since putative HRE sequences were identified within the promoter regions of HIF1A-AS1 and HIF1A-AS2, we aimed to ascertain whether HIF-1 $\alpha$ regulates HIF1A-AS1 and HIF1A-AS2 by silencing HIF-1 $\alpha$. Results demonstrated that both HIF1A-AS1 (Fig. 4D) and HIF1A-AS2 (Fig. 4E) were significantly inhibited by HIF-1 $\alpha$ siRNA $(\mathrm{P}<0.05)$, indicating that HIF-1 $\alpha$ acts as an upstream regulatory factor of HIF1A-AS1 and HIF1A-AS2.

Effects of the silencing of HIF-1 $\alpha, H I F 1 A-A S 1$ and HIF1A-AS2 on the osteogenic biomarkers of PDLCs. To further investigate the effects of HIF-1 $\alpha$, HIF1A-AS1 and HIF1A-AS2 on the osteogenic differentiation of PDLCs, we investigated the changes in Runx 2 mRNA, Runx 2 protein and ALP activity following the silencing of HIF-1 $\alpha$, HIF1A-AS1 and HIF1A-AS2 under hypoxia. The levels of osteogenic biomarkers (Fig. 5), Runx 2 protein, Runx 2 mRNA and ALP activity, were significantly inhibited by the silencing of HIF-1 $\alpha$ and induced by the silencing of HIF1A-AS2 and co-silencing of HIF1A-AS1 and HIF1A-AS2 $(\mathrm{P}<0.05)$, while there were no significant changes following the silencing of HIF1A-AS1 alone $(\mathrm{P}>0.05)$. 
A
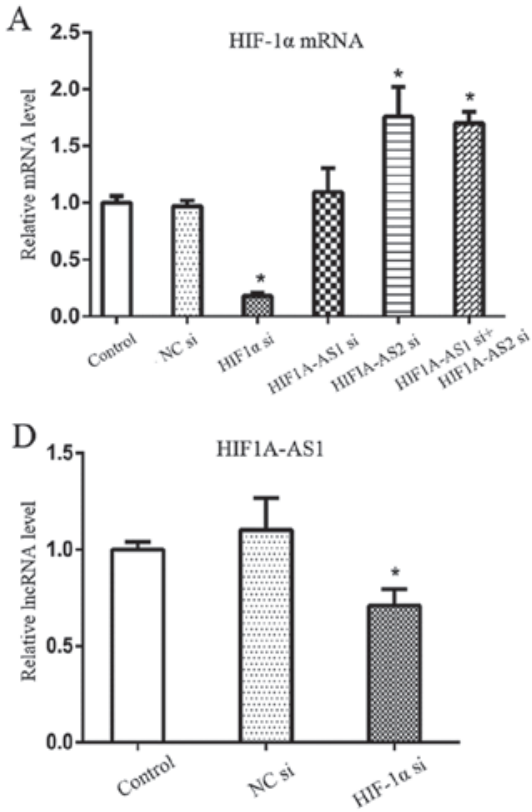

B

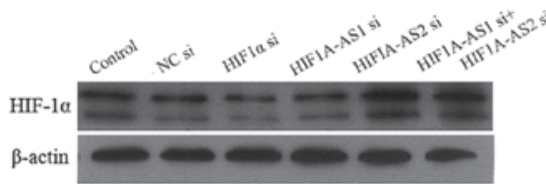

$\mathrm{E}$

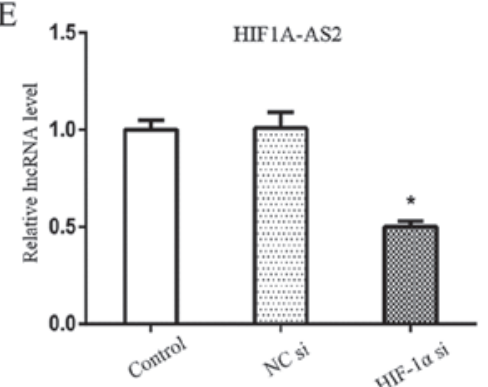

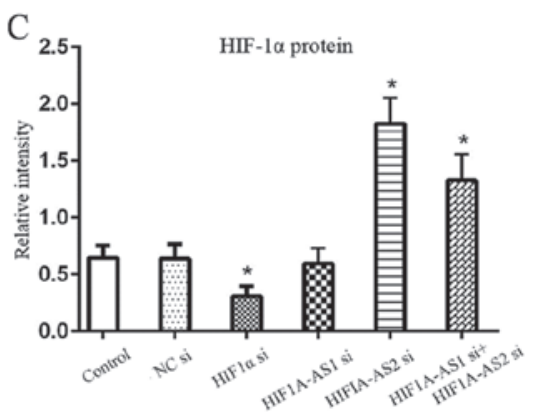

Figure 4. Effects of the silencing of HIF1A-AS1, HIF1A-AS2 and HIF-1 $\alpha$ on the expression of each other in hypoxia. (A) HIF-1 $\alpha$ mRNA showed no significant change following the silencing of HIF1A-AS1 in hypoxia, while it was significantly induced following the silencing of HIF1A-AS1 or co-silencing of HIF1A-AS1 and HIF1A-AS2. (B and C) Changes in HIF-1 $\alpha$ protein were consistent with that of HIF-1 $\alpha$ mRNA following the silencing of HIF1A-AS1 and HIF1A-AS2 in hypoxia. (D and E) HIF1A-AS1 and HIF1A-AS2 were significantly suppressed following the silencing of HIF-1 $\alpha$ in hypoxia. "P<0.05 vs. the control group, n=3. HIF1A-AS1, HIF1A antisense RNA 1; HIF1A-AS2, HIF1A antisense RNA 2; HIF-1 $\alpha$, hypoxia-inducible factor-1 $\alpha$.
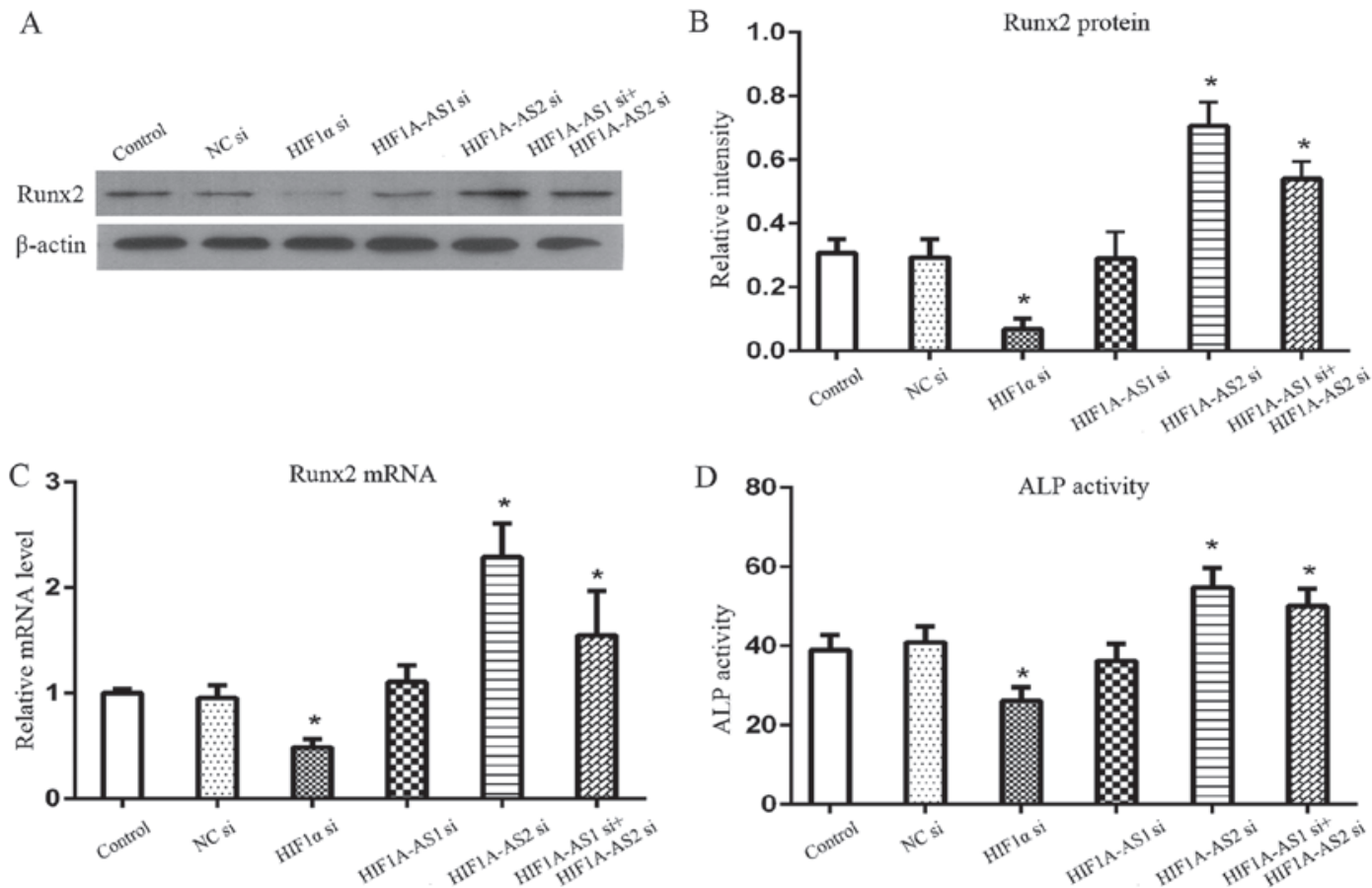

Figure 5. Effects of the silencing of HIF1A-AS1, HIF1A-AS2 and HIF-1 $\alpha$ on the expression of osteogenic biomarkers in PDLCs in hypoxia. (A and B) Silencing of HIF-1 $\alpha$ significantly inhibited Runx2 protein under hypoxia. Silencing of HIF1A-AS1 or co-silencing of HIF1A-AS1 and HIF1A-AS2 obviously promoted Runx2 protein in hypoxia, while silencing of HIF1A-AS2 alone did not significantly alter Runx2 protein. (C) Changes in the Runx2 mRNA level under different treatment conditions in hypoxia. (D) Changes in ALP activity under different treatment conditions in hypoxia. " $\mathrm{P}<0.05$ vs. the control group, $\mathrm{n}=3$. HIF1A-AS1, HIF1A antisense RNA 1; HIF1A-AS2, HIF1A antisense RNA 2; HIF-1 $\alpha$, hypoxia-inducible factor-1 $\alpha$; PDLCs, periodontal ligament cells; Runx2, runt-related transcription factor 2; ALP, alkaline phosphatase.

\section{Discussion}

The functions of HIF1A-AS1 and HIF1A-AS2 have not been studied in PDLCs. HIF1A-AS1 and HIF1A-AS2 play key roles in cell proliferation and apoptosis, and could be used as predictive biomarkers for cancers (26-29). However, little is known concerning HIF1A-AS1 and HIF1A-AS2 in periodontal tissues under hypoxia. Thus, we explored the complex relationships 
among HIF1A-AS1, HIF1A-AS2 and HIF-1 $\alpha$ and investigated whether they regulate the osteogenic differentiation of PDLCs.

In the present study, we discovered that the two antisense lncRNAs, HIF1A-AS1 and HIF1A-AS2, were expressed in PDLCs. Our present findings showed that the expression levels of HIF1A-AS1, HIF1A-AS2, HIF-1 $\alpha$ and osteogenic biomarkers were altered in a temporal manner under hypoxia. Both HIF1A-AS1 and HIF1A-AS2 were expressed at low levels under normoxia, but they were obviously induced in short-term hypoxia, reaching a peak at $12 \mathrm{~h}$. Increased HIF1A-AS2 under hypoxia was found in the first few hours by researchers in various cancer cells $(17,30)$. The expression patterns of HIF-1 $\alpha$ protein and the osteogenic markers (Runx2 and ALP activity) were consistent with that of HIF1A-AS1 and HIF1A-AS2. HIF-1 $\alpha$ mRNA was highly expressed under hypoxia and then declined gradually after $6 \mathrm{~h}$. In addition, Huang et al (31) also observed that HIF-1 $\alpha$ mRNA was elevated at $1 \mathrm{~h}$ and peaked at $6 \mathrm{~h}$ under hypoxia in human mesenchymal stem cells (hMSCs), and then the level was gradually declined from 6 to $24 \mathrm{~h}$. Uchida et al (20) found that HIF-1 $\alpha$ protein was highly induced during the first few hours in hypoxia, regulated by translation or post-translation pathways, and inhibited in prolonged hypoxia due to the decline in HIF-1 $\alpha$ mRNA. With respect to the osteogenic differentiation ability under hypoxia, Wu et al (32) demonstrated that Runx2 mRNA and protein were immediately enhanced from $1 \mathrm{~h}$ and ALP activity was from $3 \mathrm{~h}$ in PDLSCs when exposed to hypoxia, and they still remained higher than the normoxia group at $24 \mathrm{~h}$. Furthermore, Ding et al (33) found that continuous hypoxia after 3 days impaired the osteogenenic differentiation of hMSCs in hypoxia. Therefore, the above studies indicated that HIF1A-AS1, HIF1A-AS2, HIF-1 $\alpha$ and the osteogenic biomarkers were altered but time-dependently under hypoxia. Our research revealed that HIF1A-AS1, HIF1A-AS2 and HIF-1 $\alpha$ might have important roles in regulating the osteogenenic differentiation of PDLCs under hypoxia.

Furthermore, we investigated whether both HIF1A-AS1 and HIF1A-AS2 participated in regulating HIF-1 $\alpha$ mRNA by silencing HIF1A-AS1 and HIF1A-AS2. Silencing of HIF1A-AS2 prominently increased HIF-1 $\alpha$ mRNA and protein, whereas the silencing of HIF1A-AS1 did not affect HIF-1 $\alpha$ mRNA and HIF-1 $\alpha$ protein, which indicated that only HIF1A-AS2 has a strong negative regulatory function on HIF-1 $\alpha$ in PDLCs. The increase in HIF-1 $\alpha$ was slightly lower following co-transfection of HIF1A-AS1 siRNA and HIF1A-AS2 siRNA than following transfection of HIF1A-AS2 siRNA alone, which might be that the amount of HIF1A-AS2 siRNA was half when co-transfecting HIF1A-AS1 siRNA $(2.5 \mu \mathrm{l})$ and HIF1A-AS2 siRNA (2.5 $\mu \mathrm{l})$. In previous studies, HIF1A-AS2 has been reported to be complementary on at least 1,027 nt of the HIF-1 $\alpha$ mRNA 3'UTR which was rich in AU elements and HIF1A-AS2 could expose these AU rich elements to accelerate the degradation of HIF-1 $\alpha$ mRNA (21). Kumar and Carmichael (34) speculated that HIF1A-AS2 suppressed HIF-1 $\alpha$ mRNA translation by hybridizing to HIF-1 $\alpha$ mRNA 3'UTR, therefore HIF-1 $\alpha$ protein was suppressed. Our bioinformatic analysis revealed that both HIF1A-AS1 and HIF1A-AS2 could be strictly complementary to HIF-1 $\alpha$ mRNA, in which HIF1A-AS1 was complementary upon $117 \mathrm{nt}$ and HIF1A-AS2 was 1,221 nt. In addition, our experiment in vitro proved that only HIF1A-AS2 significantly downregu- lated HIF-1 $\alpha$. By analyzing the regulatory mechanism of the HIF-system in human macrophages, elevated HIF-1 $\alpha$ mRNA and protein were also observed when silencing HIF1A-AS2, which was in accordance with our results (35). Bertozzi et al (19) also reported that HIF1A-AS1 and HIF1A-AS2 had different responses to different stimulations. Since HIF1A-AS1 accumulates at the nuclear membrane, it might be associated with the export of mRNA from the nucleus into the cytoplasm. Therefore, we predicted that HIF1A-AS1 and HIF1A-AS2 may be involved in different types of regulatory mechanisms.

HIF-1 was speculated as the upstream factor of the antisense HIF-1 $\alpha$. Some evidence was provided by Uchida et al (20) to support this hypothesis. i) Cycloheximide, a protein synthesis inhibitor that restrains hypoxia-induced HIF-1 $\alpha$ increase, also inhibited the augmentation of HIF1A-AS2 during long-term hypoxia; ii) HIF-1 $\alpha$ was found to be bound to the putative HRE sequence, which could be displaced by the oligonucleotide sequence of HRE found in human HIF1A-AS2 gene promoter, not by a mismatch of putative HRE sequence. Moreover, in embryonic cells lacking the HIF- $\beta$ subunit where HIF-1 could not be formed, hypoxia-induced HIF1A-AS2 was attenuated, indicating that HIF1A-AS2 was HIF-1 responsive $(36,37)$. Consistently, the HIF1A-AS1 promoter also has putative HREs. To further investigate the effects of HIF-1 $\alpha$ on HIF1A-AS1 and HIF1A-AS2, we silenced HIF-1 $\alpha$, and the results showed that HIF1A-AS1 and HIF1A-AS2 were downregulated, which confirmed that HIF-1 $\alpha$ has a positive regulatory effect on both HIF1A-AS1 and HIF1A-AS2. This was also consistent with the observation that HIF1A-AS1 and HIF1A-AS2 were decreased after $12 \mathrm{~h}$ under hypoxia accompanied by the decline of HIF-1 $\alpha$ protein.

HIF1A-AS1 and HIF1A-AS2 also had different roles in regulating the osteogenic differentiation of PDLCs under hypoxia, which might be related to HIF-1 $\alpha$. Inhibition of HIF-1 $\alpha$ significantly suppressed osteogenic biomarkers, which demonstrated that HIF-1 $\alpha$ positively regulated the osteogenic differentiation ability of PDLCs. Typically, HIF-1 $\alpha$-induced downstream proteins respond rapidly, usually occurring during the first several hours $(17,38)$. Zhou et al $(32,39)$ found that hypoxia-simulated osteogenesis was via ERK1/2 and p38-mediated HIF-1 $\alpha$ pathway, in which the ERK1/2 pathway was the dominant one. ERK1/2 and p38, members of the MAPKs, were found to be involved not only in maintaining the stability and promoting the transactivation of HIF- $1 \alpha$, but also in regulating osteogenic differentiation. Furthermore, we found that silencing of HIF1A-AS2 significantly promoted the expression of osteogenic biomarkers, whereas silencing of HIF1A-AS1 had no significant effects on the expressions of osteogenic biomarkers. It should be noted that the changes in the osteogenic biomarkers were in accordance with the changes in HIF-1 $\alpha$ following the silencing of HIF1A-AS2, since HIF-1 $\alpha$ regulated the osteogenic differentiation of PDLCs. Therefore, we speculated that there may be an HIF1A-AS2/HIF-1 $\alpha$ signaling pathway through which to regulate the osteogenic differentiation of PDLCs.

In conclusion, we found that both HIF1A-AS1 and HIF1A-AS2 were complementary to HIF-1 $\alpha$ mRNA by bioinformatic analysis, but the in vitro study revealed that only HIF1A-AS2 had an inhibitory effect on HIF-1 $\alpha$ in PDLCs. In addition, HIF-1 $\alpha$ also induced HIF1A-AS1 and HIF1A-AS2, which have putative HREs in the promoters. Furthermore, 
HIF-1 $\alpha$ promoted the osteogenic differentiation of PDLCs and HIF1A-AS2 inhibited the osteogenic differentiation of PDLCs.

\section{Acknowledgements}

The present study was supported by the Natural Science Foundation of Guangdong Province (no. 2015A030313083) and the Natural Science Foundation of China (no. 81400499).

\section{References}

1. Hong JW, Noh JH and Kim DJ: The prevalence and associated factors of periodontitis according to fasting plasma glucose in the Korean adults: The 2012-2013 Korea National Health and Nutrition Examination Survey. Medicine (Baltimore) 95: e3226, 2016.

2. Eke PI, Zhang X, Lu H, Wei L, Thornton-Evans G, Greenlund KJ, Holt JB and Croft JB: Predicting periodontitis at state and local levels in the United States. J Dent Res 95: 515-522, 2016.

3. Eke PI, Dye BA, Wei L, Slade GD, Thornton-Evans GO, Borgnakke WS, Taylor GW, Page RC, Beck JD and Genco RJ: Update on prevalence of periodontitis in adults in the United States: NHANES 2009 to 2012. J Periodontol 86: 611-622, 2015.

4. Niklas A, Proff P, Gosau M and Römer P: The role of hypoxia in orthodontic tooth movement. Int J Dent 2013: 841840, 2013.

5. Hellwig-Bürgel T, Stiehl DP, Wagner AE, Metzen E and Jelkmann W: Review: Hypoxia-inducible factor-1 (HIF-1): A novel transcription factor in immune reactions. J Interferon Cytokine Res 25: 297-310, 2005.

6. Semenza GL and Wang GL: A nuclear factor induced by hypoxia via de novo protein synthesis binds to the human erythropoietin gene enhancer at a site required for transcriptional activation. Mol Cell Biol 12: 5447-5454, 1992.

7. Nallamshetty S, Chan SY and Loscalzo J: Hypoxia: A master regulator of microRNA biogenesis and activity. Free Radic Biol Med 64: 20-30, 2013.

8. Wang XJ and Si LB: Advances on hypoxia inducible factor-1. Chin Med J (Engl) 126: 3567-3571, 2013.

9. Holzwarth C, Vaegler M, Gieseke F, Pfister SM, Handgretinger R, Kerst $\mathrm{G}$ and Müller I: Low physiologic oxygen tensions reduce proliferation and differentiation of human multipotent mesenchymal stromal cells. BMC Cell Biol 11: 11, 2010.

10. Yang DC, Yang MH, Tsai CC, Huang TF, Chen YH and Hung SC: Hypoxia inhibits osteogenesis in human mesenchymal stem cells through direct regulation of RUNX2 by TWIST. PLoS One 6: e23965, 2011.

11. Zhou Y, Fan W and Xiao Y: The effect of hypoxia on the stemness and differentiation capacity of PDLC and DPC. BioMed Res Int 2014: 890675, 2014.

12. Zou D, Han W, You S, Ye D, Wang L, Wang S, Zhao J, Zhang W, Jiang X, Zhang X, et al: In vitro study of enhanced osteogenesis induced by HIF-1 $\alpha$-transduced bone marrow stem cells. Cell Prolif 44: 234-243, 2011.

13. Masoud GN and Li W: HIF-1 $\alpha$ pathway: Role, regulation and intervention for cancer therapy. Acta Pharm Sin B 5: 378-389, 2015.

14. Parsanejad M, Zhang Y, Qu D, Irrcher I, Rousseaux MW, Aleyasin H, Kamkar F, Callaghan S, Slack RS, Mak TW, et al: Regulation of the VHL/HIF-1 pathway by DJ-1. J Neurosci 34: 8043-8050, 2014.

15. Richard DE, Berra E, Gothié E, Roux D and Pouysségur J: p42/p44 mitogen-activated protein kinases phosphorylate hypoxia-inducible factor 1alpha (HIF-1alpha) and enhance the transcriptional activity of HIF-1. J Biol Chem 274: 32631-32637, 1999.

16. Zhong H, Chiles K, Feldser D, Laughner E, Hanrahan C, Georgescu MM, Simons JW and Semenza GL: Modulation of hypoxia-inducible factor 1alpha expression by the epidermal growth factor/phosphatidylinositol 3-kinase/PTEN/AKT/FRAP pathway in human prostate cancer cells: implications for tumor angiogenesis and therapeutics. Cancer Res 60: 1541-1545, 2000.

17. Thrash-Bingham CA and Tartof KD: aHIF: A natural antisense transcript overexpressed in human renal cancer and during hypoxia. J Natl Cancer Inst 91: 143-151, 1999.

18. Baranello L, Bertozzi D, Fogli MV, Pommier Y and Capranico G: DNA topoisomerase I inhibition by camptothecin induces escape of RNA polymerase II from promoter-proximal pause site, antisense transcription and histone acetylation at the human HIF-1alpha gene locus. Nucleic Acids Res 38: 159-171, 2010.
19. Bertozzi D, Iurlaro R, Sordet $\mathrm{O}$, Marinello J, Zaffaroni $\mathrm{N}$ and Capranico G: Characterization of novel antisense HIF-1 $\alpha$ transcripts in human cancers. Cell Cycle 10: 3189-3197, 2011.

20. Uchida T, Rossignol F, Matthay MA, Mounier R, Couette S, Clottes E and Clerici C: Prolonged hypoxia differentially regulates hypoxia-inducible factor (HIF)-1alpha and HIF-2alpha expression in lung epithelial cells: implication of natural antisense HIF-1alpha. J Biol Chem 279: 14871-14878, 2004.

21. Rossignol F, Vaché C and Clottes E: Natural antisense transcripts of hypoxia-inducible factor 1alpha are detected in different normal and tumour human tissues. Gene 299: 135-140, 2002.

22. Komori T: Regulation of osteoblast differentiation by Runx2. Adv Exp Med Biol 658: 43-49, 2010.

23. Bhargavan B, Gautam AK, Singh D, Kumar A, Chaurasia S, Tyagi AM, Yadav DK, Mishra JS, Singh AB, Sanyal S, et al: Methoxylated isoflavones, cajanin and isoformononetin, have non-estrogenic bone forming effect via differential mitogen activated protein kinase (MAPK) signaling. J Cell Biochem 108: 388-399, 2009.

24. Liu Q, Geng H, Xue C, Beer TM and Qian DZ: Functional regulation of hypoxia inducible factor- $1 \alpha$ by SET9 lysine methyltransferase. Biochim Biophys Acta 1853: 881-891, 2015.

25. Rhim T, Lee DY and Lee M: Hypoxia as a target for tissue specific gene therapy. J Control Release 172: 484-494, 2013.

26. Chen WM, Huang MD, Kong R, Xu TP, Zhang EB, Xia R, Sun M, De W and Shu YQ: Antisense long non-coding RNA HIF1A-AS2 is upregulated in gastric cancer and associated with poor prognosis. Dig Dis Sci 60: 1655-1662, 2015.

27. He Q, Tan J, Yu B, Shi W and Liang K: Long non-coding RNA HIF1A-AS1A reduces apoptosis of vascular smooth muscle cells: Implications for the pathogenesis of thoracoabdominal aorta aneurysm. Pharmazie 70: 310-315, 2015.

28. Tantai J, Hu D, Yang Y and Geng J: Combined identification of long non-coding RNA XIST and HIF1A-AS1 in serum as an effective screening for non-small cell lung cancer. Int J Clin Exp Pathol 8: 7887-7895, 2015.

29. Wang S, Zhang X, Yuan Y, Tan M, Zhang L, Xue X, Yan Y, Han L and Xu Z: BRG1 expression is increased in thoracic aortic aneurysms and regulates proliferation and apoptosis of vascular smooth muscle cells through the long non-coding RNA HIF1A-AS1 in vitro. Eur J Cardiothorac Surg 47: 439-446, 2015.

30. Rossignol F, de Laplanche E, Mounier R, Bonnefont J, Cayre A, Godinot C, Simonnet H and Clottes E: Natural antisense transcripts of HIF-lalpha are conserved in rodents. Gene 339: 121-130, 2004.

31. Huang J, Deng F, Wang L, Xiang XR, Zhou WW, Hu N and Xu L: Hypoxia induces osteogenesis-related activities and expression of core binding factor $\alpha 1$ in mesenchymal stem cells. Tohoku J Exp Med 224: 7-12, 2011.

32. Wu Y, Yang Y, Yang P, Gu Y, Zhao Z, Tan L, Zhao L, Tang T and Li Y: The osteogenic differentiation of PDLSCs is mediated through MEK/ERK and p38 MAPK signalling under hypoxia. Arch Oral Biol 58: 1357-1368, 2013.

33. Ding H, Chen S, Yin JH, Xie XT, Zhu ZH, Gao YS and Zhang CQ: Continuous hypoxia regulates the osteogenic potential of mesenchymal stem cells in a time-dependent manner. Mol Med Rep 10: 2184-2190, 2014.

34. Kumar M and Carmichael GG: Nuclear antisense RNA induces extensive adenosine modifications and nuclear retention of target transcripts. Proc Natl Acad Sci USA 94: 3542-3547, 1997.

35. Poitz DM, Augstein A, Hesse K, Christoph M, Ibrahim K, Braun-Dullaeus RC, Strasser RH and Schmeißer A: Regulation of the HIF-system in human macrophages - differential regulation of HIF- $\alpha$ subunits under sustained hypoxia. Mol Immunol 57: 226-235, 2014

36. Maltepe E, Schmidt JV, Baunoch D, Bradfield CA and Simon MC: Abnormal angiogenesis and responses to glucose and oxygen deprivation in mice lacking the protein ARNT. Nature 386: 403-407, 1997.

37. Neckers LM: aHIF: The missing link between HIF-1 and VHL? J Natl Cancer Inst 91: 106-107, 1999.

38. Wang GL, Jiang BH, Rue EA and Semenza GL: Hypoxia-inducible factor 1 is a basic-helix-loop-helix-PAS heterodimer regulated by cellular $\mathrm{O}_{2}$ tension. Proc Natl Acad Sci USA 92: 5510-5514, 1995.

39. Zhou Y, Guan X, Wang H, Zhu Z, Li C, Wu S and Yu H: Hypoxia induces osteogenic/angiogenic responses of bone marrow-derived mesenchymal stromal cells seeded on bone-derived scaffolds via ERK1/2 and p38 pathways. Biotechnol Bioeng 110: 1794-1804, 2013. 